УДК 661.862`022:54-414

\title{
Effective Separation of Fullerene Mixture by Sorbent on The Base of Aluminium Oxide
}

\author{
Irina A. Dubinina ${ }^{\mathrm{a}, \mathrm{b}}$, Vladislav A. Lopatin ${ }^{\mathrm{b}}$, \\ Gariy A. Glushenko ${ }^{\text {b }}$, Natalia G. Vnukova ${ }^{\mathrm{a}, \mathrm{b}}$, \\ Anatoly M. Zhyzhaev ${ }^{c}$, Yevgeny V. Tomashevich', \\ Grigory N. Churilov and Eugenia M. Kuzmina ${ }^{a, b}$ \\ ${ }^{a}$ Siberian Federal University \\ 79 Svobodny, Krasnoyarsk, 660041, Russia \\ ${ }^{b}$ L.V. Kirensky Institute of Physics SB RAS \\ 50/38 Akademgorodok, Krasnoyarsk, 660036, Russia \\ 'Insitute of Chemistry and Chemistry Technology SB RAS \\ 50/24 Akademgorodok, Krasnoyarsk, 660036, Russia
}

Received 21.09.2015, received in revised form 03.10.2015, accepted 25.11.2015

In this paper the results of synthesis and investigate of novel sorbent on the base of aluminium oxide with carbon cover are presented. It was established that application of concentrate fullerene solution on the oxide aluminium powder and following annealing allowed to synthesize sorbent with the surface which is density cover by carbon. The structure of aluminium oxide after annealing does not change significantly according to RFCA data. It was established by XPS method that molecules of $C_{60}$ chemically bonded with aluminium oxide are presented at the carbon cover. There are oxygencontaining groups and amorphous carbon on the sorbent surface. The test of using sorbet show the effective extraction of $C_{60}$ and $C_{70}$ from the fullerene mixture by single stage separation: $C_{60}$ with purity $98 \%$ and $C_{70}$ with purity $70 \%$.

Keywords: fullerene, liquid column chromatography, aluminium oxide.

DOI: 10.17516/1998-2836-2015-8-4-620-625.

(C) Siberian Federal University. All rights reserved

* Corresponding author E-mail address: churilov@iph.krasn.ru 


\title{
Эффективное разделение фуллереновой смеси сорбентом
}

\section{на основе оксида алюминия}

\author{
И.А. Дубинина ${ }^{\mathrm{a}, \boldsymbol{\sigma}}$, В.А. Лопатин, \\ Г.А. Глущенко ${ }^{\sigma}$, Н.Г. Внукова ${ }^{a, \tilde{0}}$, А.М. Жижаев ${ }^{\mathrm{B}}$, \\ Е.В. Томашевич ${ }^{\mathrm{B}}$, Е.М. Кузьмина ${ }^{\mathrm{a}}$, Г.Н. Чурилов ${ }^{\mathrm{a}, \tilde{\sigma}}$ \\ ${ }^{a}$ Сибирский федеральный университет \\ Россия, 660041, Красноярск, пр. Свободный, 79 \\ ${ }^{6}$ Институт физики им. Л.В. Киренского СО РАН \\ Россия, 660036, Красноярск, Академгородок, 50/38 \\ ${ }^{6}$ Институт химии и химической технологии СО РАН \\ Россия, 660036, Красноярск, Академгородок, 50/24
}

В работе представлень результаты получения и исследования нового сорбента на основе оксида алюминия, покрытого углеродной оболочкой. Установлено, что нанесение на порошок оксида алюминия концентрированного раствора фуллеренов и последующиий отжиг позволяет получить сорбент, поверхность которого плотно порыта углеродной оболочкой. Данные РФА показывают, что структура оксида алюминия после отжига значительно не меняется. Методом ФЭС установлено, что в углеродной оболочке присутствуют молекуль фуллерена $C_{60}$, химически связанные с оксидом алюминия. На поверхности сорбента имеются кислородсодержащие группы, аморфный углерод. Испытания сорбента на образие фуллереновой смеси продемонстрировали эффективное выделение $C_{60}$ u $C_{70}$ уже после однократного разделения: фракциии $C_{60}$ чистотой $98 \%$ и $C_{70}$ чистотой $70 \%$.

Ключевые слова: фуллерены, хроматография, оксид алюминия.

\section{Введение}

Уникальная структура как фуллеренов, так и эндоэдральных фуллеренов, содержащих различные атомы металлов, в силу уникальности проявляемых этими веществами свойств вызывают интерес не только с фундаментальной точки зрения, но и с прикладной [1]. Однако и на сегодняшний день вопрос эффективного производства индивидуальных фуллеренов все еще остается нерешенным. Как показали многочисленные исследования, в качестве стационарной фазы при разделении фуллереновой смеси методом жидкостной хроматографии лучше всего применять углеродные соединения: графиты, сажи и угли [2]. У перечисленных сорбентов имеется один большой недостаток: при многократном использовании эффективность разделения падает вследствие их низкой механической прочности. На текущий момент разделение выполняют путем применения сорбентов с твердой основой и привитой фазой, например Buckyprep, методом высокоэффективной жидкостной хроматографии. Однако такие сорбенты очень чувствительны к экстрагентам фуллереновой смеси, поэтому не всегда подходят для перпаративного выделения индивидуальных фуллеренов [3]. Оптимальный сорбент для разделе-

$$
-621-
$$


ния фуллереновой смеси на индивидуальные фуллерены методом жидкостной хроматографии не найден.

В данной работе мы приводим результаты исследования полученного нами сорбента на основе $\mathrm{Al}_{2} \mathrm{O}_{3}$ с нанесенной на него графитовой оболочкой.

\section{Обсуждение и результаты}

В качестве твердой основы мы использовали порошок $\mathrm{Al}_{2} \mathrm{O}_{3}$ для хроматографии. Сорбент был изготовлен путем капельного нанесения концентрированного раствора фуллереновой смеси (состава: $\mathrm{C}_{60}\left(70 \% \%_{\text {вес }}\right), \mathrm{C}_{70}(25 \%$ вес $)$ и $\mathrm{C}_{\mathrm{n}}$ при $\mathrm{n}>70(5 \%$ вес $\left.)\right)$ на порошок с последующим высушиванием и отжигом при температуре $800^{\circ} \mathrm{C}$ в герметичном стальном цилиндре с прокладкой из отожженной меди. На рис. 1 представлены изображения исходного оксида алюминия (a) и отожженного (б), полученные на сканирующем электронном микроскопе ТМ-1000. Можно видеть, что углерод покрывает частицы оксида алюминия плотным слоем.

Исследования рентгенофазовым методом показали [4], что термическая обработка не привела к существенным изменениям структуры оксида алюминия, рис. 2.

Методом фотоэлектронной спектроскопии было установлено, что в полученном порошке присутствуют связи алюминия с $\mathrm{C}_{60}$, С-C (в молекуле $\mathrm{C}_{60}$ ), $\mathrm{C}=\mathrm{O}, \mathrm{C}-\mathrm{O}-\mathrm{C}$, связи, характерные для аморфного углерода, рис. 3. Те энергии связи алюминия, которые были нами определены, однозначно соответствуют связям алюминия именно с этими соединениями в соответствии с литературными данными [5].

Нами было выполнено исследование сорбционных характеристик полученного сорбента. Определение удельной поверхности образцов проводилось методом тепловой десорбции азота по стандартной методике. Значения удельной поверхности, размера и объема пор были рассчитаны по БЭТ и составили $118,29 \mathrm{M}^{2} / \Gamma ; 0,254 \mathrm{~cm}^{3} / \Gamma$ и 8,6 нм, соответственно. Определение предельного объема сорбционного пространства $\left(\mathrm{W}_{\mathrm{s}}\right)$ проводилось по следующей методике: предварительная десорбция и последующее насыщение образцов парами органического растворителя проводилось в специальных калиброванных по массе стеклянных мини-бюксах с пришлифованными крышками. Навеску образца загружали в бюкс, помещали в сушильный шкаф и, подняв температуру до $100-120{ }^{\circ} \mathrm{C}$, выдерживали
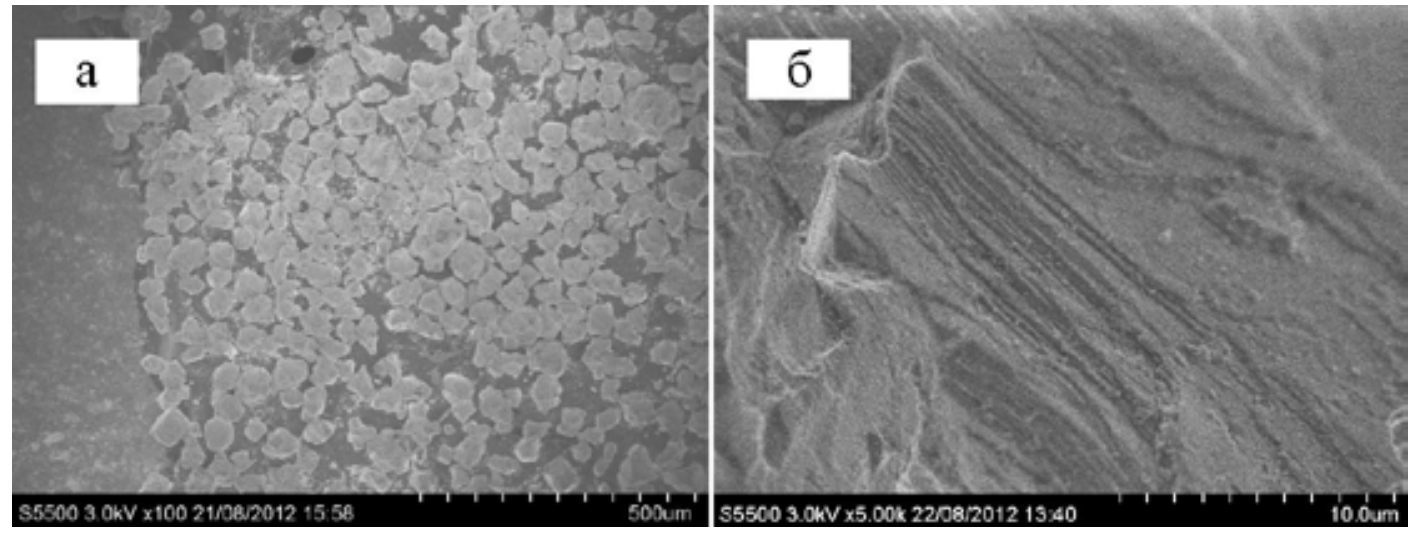

Рис. 1. Микрофотографии исходного (а) и отожженного $\mathrm{Al}_{2} \mathrm{O}_{3}$ 


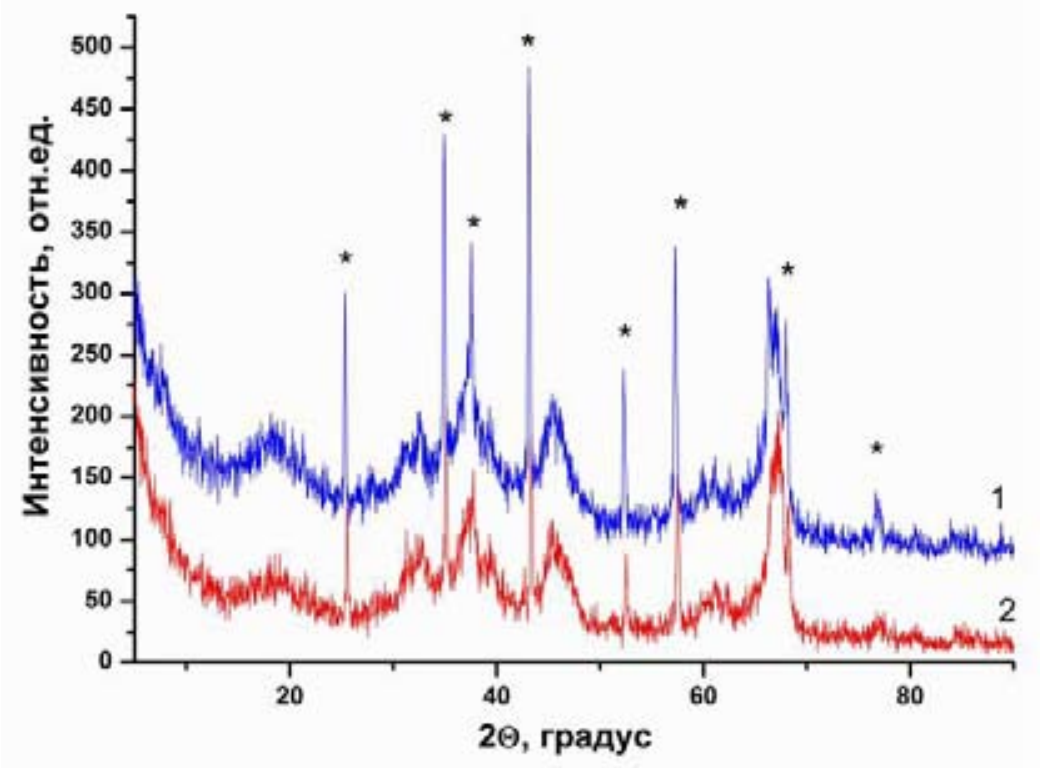

Рис. 2. Рентгенограмма графитизированного порошка $\mathrm{Al}_{2} \mathrm{O}_{3}: 1$ - до отжига; 2 - после отжига

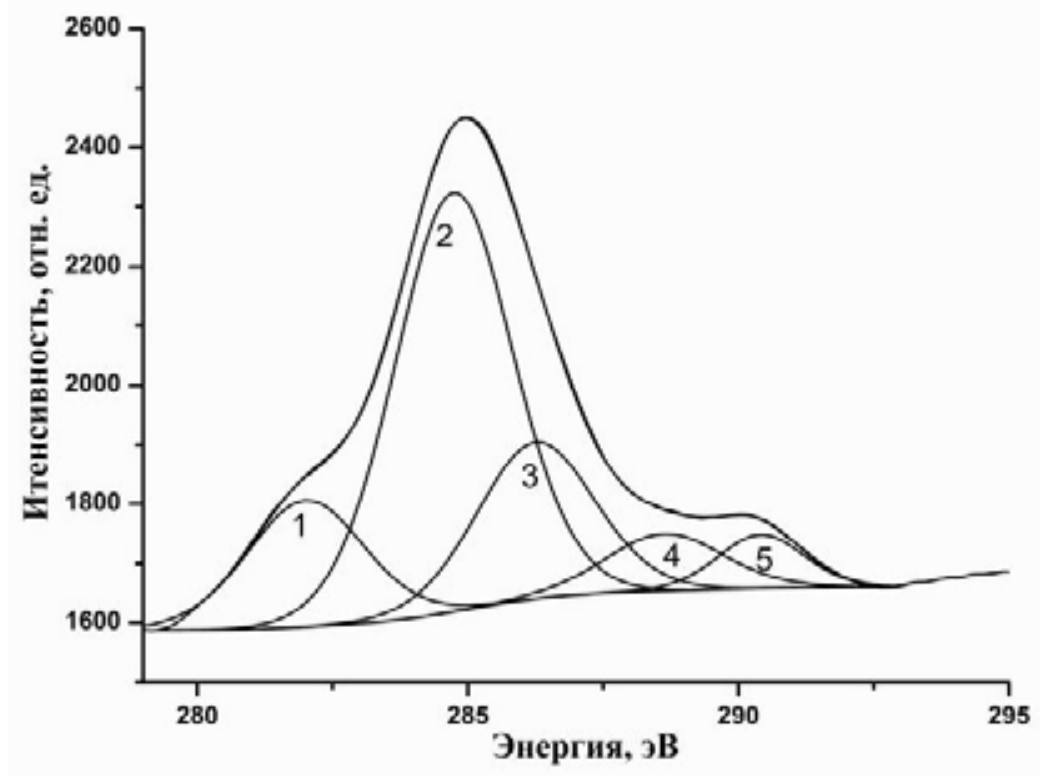

Рис. 3. 1s-линия углерода: 1 - 282 эВ Al-C; 2 - 284,7 эВ С-С ( в молекуле $\mathrm{C}_{60}$ ); 3 - 286,2 эВ sp гибридизированный углерод; 4 - 288,6 эВ C=O; 5 - 290,4 эВ С-O-C

в течение 2 ч; герметически плотно закрывали бюкс крышкой и, охладив его до комнатной температуры, взвешивали на аналитических весах $\left(\mathrm{m}_{1}\right)$. Затем быстро снимали крышку бюкса и помещали его в эксикатор, на дне которого находился жидкий сорбат. Насыщение образца парами сорбата осуществлялось в течение заданного времени. После этого извлекали бюкс с образцом из эксикатора, быстро закрывали его крышкой и взвешивали 
на аналитических весах, определяя таким образом $\mathrm{m}_{2}$. Величина сорбции определяется по результатам взвешивания как

$$
\mathrm{Ws}=\left(\mathrm{m}_{2}-\mathrm{m}_{1}\right) /\left(\mathrm{m}_{1}-\mathrm{m}\right),
$$

где $\mathrm{m}$ - масса пустого бюкса.

Результаты измерений представлены в табл. 1.

Функциональные группы на поверхности сорбента определяли потенциометрическим титрованием по методу Бёма. Нейтрализация кислых поверхностных групп проводилась тремя основаниями различной силы: $\mathrm{NaOH}$ (титруются карбоксильные, фенольные и лактонные группы), $\mathrm{Na}_{2} \mathrm{CO}_{3}$ (лактонные и карбоксильные) и $\mathrm{NaHCO}_{3}$ (карбоксильные). Результаты измерений представлены в табл. 2.

Полученный и аттестованный сорбент был испытан в качестве стационарной фазы для жидкостного хроматографического разделения пробы фуллереновой смеси. В качестве элюента была использована смесь толуол-гексан в соотношении 1:4. В результате разделения получены две фракции, которые были проанализированы методом HPLC на хроматографе Agilent 1200 Series, укомплектованном колонкой Buckyprep.

Первая выделенная фракция соответствует фуллерену $\mathrm{C}_{60}$ чистотой 98 \% (определена по отношению площади пика $\mathrm{C}_{60}$ к общей сумме площадей всех пиков) (рис. 4). Вторая фракция соответствует фуллерену $\mathrm{C}_{70}$ чистотой 70 \% (определена по отношению площади пика $\mathrm{C}_{70} \mathrm{~K}$ общей сумме площадей всех пиков).

\section{Выводы}

Таким образом, в работе показано, что нанесение фуллереновой пленки на поверхность оксида алюминия и последующая термическая обработка порошка позволяют получать сорбент, разделяющий фуллерены с высоким разрешением, и продемонстрирована возможность его применения в жидкостной колоночной хроматографии.

Таблица 1. Сорбционная емкость порошков по парам органических растворителей

\begin{tabular}{|l|c|c|c|c|c|c|c|}
\hline Растворитель & Гексан & Бензол & Толуол & Этанол & О-ксилол & Пиридин & Ацетон \\
\hline $\mathrm{W}_{\mathrm{s}}, \mathrm{M \Gamma} / \Gamma$ & 0.254 & 0.402 & 0.259 & 0.195 & 0.217 & 0.381 & 0.239 \\
\hline
\end{tabular}

Таблица 2. Количество кислых групп на поверхности сорбента

\begin{tabular}{|c|c|}
\hline Группы & Количество, ммоль/г \\
\hline Общее число групп & 1.43 \\
\hline Карбоксильные группы & 0.91 \\
\hline Лактонные группы & 0.15 \\
\hline Фенольные группы & 0.37 \\
\hline
\end{tabular}




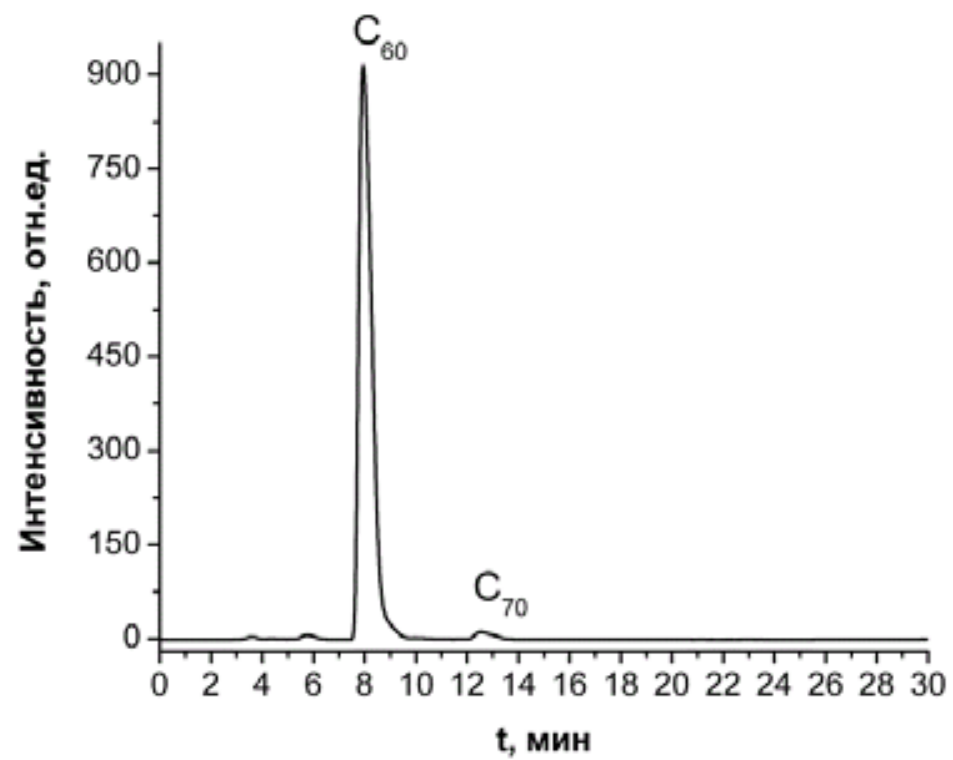

Рис. 4. Хроматограмма фракции $\mathrm{C}_{60}$, время выхода 7,9 мин

Работа выполнена при поддержке гранта ФЦП Министерства образования и науки РФ.- Соглашение № 14.613.21.0010, ID RFMEFI61314X0010.

\section{Список литературы}

1. Popov A.A., Yang S., Dunsch L. Endohedral Fullerenes. Chemical Reviews 2013. Vol. 113(8), P. 5989-6113.

2. Taylor R., Hare J.P., Abdul-Sada A.K., Kroto H.W. Isolation, separation and characterisation of the fullerenes $\mathrm{C}_{60}$ and $\mathrm{C}_{70}$ : The third form of carbon. Journal of the Chemical Society, Chemical Communications 1990. (20), P. 1423-1425.

3. Jovanovic T., Koruga D., Jovancicevic B., Simic-Krstic J. Modifications of fullerenes extractions and chromatographies with different solvents. Fullerenes Nanotubes and Carbon Nanostructures 2003. Vol. 11(4), P. 383-394.

4. Botos Á., Khlobystov A.N., Botka B., HacklR., Székely E., Simándi B. Kamarás K. Investigation of fullerene encapsulation in carbon nanotubes using a complex approach based on vibrational spectroscopy. Physica Status Solidi B 2010. Vol. 247(11-12), P. 2743-2745.

5. Popov M., Medvedev V. Fullerid of aluminum nanoclasters. Journal of Applied Physics 2010. Vol. 108, P. 094317-1-094317-6. 